\title{
Effect of experience with a preferred food on consummatory responding for a less preferred food in goldfish
}

\author{
P. A. COUVILLON and M. E. BITTERMAN \\ University of Hawaii, Honolulu, Hawaii
}

\begin{abstract}
In two experiments with goldfish patterned after work with rats, consummatory responding for a less preferred food (adulterated with quinine) was measured in subjects that did or did not have previous experience of a preferred (unadulterated) food. The result in each case was not the negative contrast found in rats, but the opposite-more responding in subjects with experience of both foods. The experiments are in accord with a variety of others that have failed to show control of performance by remembered reward in goldfish.
\end{abstract}

As Flaherty (1982) noted in a recent review of the extensive literature on incentive contrast, the successive negative-contrast effect discovered in rats by Elliott (1928) has failed repeatedly to appear in experiments with goldfish (Gonzalez, Ferry, \& Powers, 1974; Gonzalez, Potts, Pitcoff, \& Bitterman, 1972; Lowes \& Bitterman, 1967; Mackintosh, 1971). When rats are trained with a preferred reward and then shifted to a less preferred reward, they commonly perform more poorly, at least for a time, than do control animals trained from the outset with the less preferred reward, but the performance of shifted goldfish does not fall below the control level. Successive negative contrast has also failed repeatedly to appear in experiments with toads (Schmajuk, Segura, \& Ruidiaz, 1981), with painted turtles (Pert \& Bitterman, 1970; Pert $\&$ Gonzalez, 1974), and with very young rats (Chen, Gross, \& Amsel, 1981; Stanton \& Amsel, 1980). Goldfish (Burns, Woodard, Henderson, \& Bitterman, 1974; Gonzalez \& Powers, 1973) and painted turtles (Pert \& Gonzalez, 1974) do, however, show simultaneous negative contrast, another contrast effect discovered in rats (Bower, 1961). Animals trained with a preferred reward for response to one of two stimuli and with a less preferred reward for response to the other stimulus perform more poorly for the less preferred reward than do control animals trained with the less preferred reward for response to both stimuli. In ontogenetic work with rats, the simultaneous effect appears before the successive (Stanton, Lobaugh, \& Amsel, 1984).

Although these results may suggest that the mechanisms of simultaneous and successive contrast are different (Bitterman, 1975; Rashotte, 1979), they do not exclude the

\footnotetext{
This research was supported in part by Grant MH33077 from the National Institute of Mental Health. We are indebted to Doris Ammon for her assistance in the collection of data and to Abram Amsel for his comments on a preliminary draft of the paper. Requests for reprints should be addressed to the Békésy Laboratory of Neurobiology, 1993 EastWest Road, Honolulu, HI 96822.
}

possibility of a common mechanism that simply is better developed in some animals than in others and is more readily expressed in some circumstances than in others. Just such an interpretation of the ontogenetic data has been offered by Stanton, Lobaugh, and Amsel (1984). According to Amsel's frustration theory, an animal that experiences the preferred reward in a training situation comes in some sense to expect it, and is frustrated upon encountering the less preferred reward instead; the frustration tends to be conditioned to sensory antecedents of the less preferred reward and therefore to interfere with subsequent instrumental performance. If frustration is better conditioned in simultaneous experiments (where presentations of the preferred and less preferred rewards are intermixed) than in successive experiments (where, typically, there is but a single transition from the preferred to the less preferred reward), and if the frustrationconditioning mechanism is not very well developed in young rats, they may be expected to show simultaneous, but not successive, contrast. Especially persuasive is the evidence that certain responses are more sensitive than others to contrast effects: 17-day-old rats trained in a runway with milk versus dry suckling (less preferred) as reward show successive negative contrast in latency of nipple-attachment after reaching the goalbox-where conditioned frustration should be strong-but not in speed of running to the goalbox. Perhaps successive negative contrast has not yet been found in goldfish because conditioned frustration in goldfish is as weak as it has been assumed to be in young rats, and because the responses measured-directed swimming and target-striking-are not very sensitive to frustration.

Whenever a phenomenon that is well known in one species fails to appear in another, the first question to be asked, of course, is whether the experiments are comparable in all important aspects (Bitterman, 1975). Because there is no way to equate the conditions of training, we have no alternative in continuing to search for the phenomenon in the latter species but to vary conditions 
widely, being guided where possible by what is known about conditions that favor the appearance of the phenomenon in the former species. In the experiments reported here, we made several substantial changes in procedure, one of which was to use rewards that differed in quality rather than in quantity. In previous goldfish experiments, differences in quantity of reward were used (for example, 1 vs. 40 Tubifex worms). This has been common practice in rat experiments, although strong successive contrast has been found as well in rats trained with rewards differing in quality (bran mash vs. sunflower seeds in Elliott's original work, 1928). Our decision to use a qualitative difference here was based on the intuition that quality might be more readily remembered. Another change was that the experimental animals were repeatedly exposed to both rewards, a procedure which might be thought to promote the development of conditioned frustration or at least to provide further opportunity for comparison. There was always, however, an interval of at least 1 day between experience with the preferred reward and experience with the less preferred reward, a precaution taken to rule out nonassociative (sensory and motivational) effects that may conceivably play some role in conventional (massed-trials) experiments on simultaneous contrast. A third change was in the measure of performance, which here was consummatory responding. We were led to a consummatory measure by the results of a set of runway experiments with rats in which sucrose solutions of different concentration were used as rewards (Flaherty, 1982): The rats showed simultaneous negative contrast in both running and drinking, but successive negative contrast only in drinking.

\section{EXPERIMENT 1}

In this experiment, individual goldfish were given daily access to one of two liquid foods-either a preferred food or the same food adulterated with quinine sulfate-and their consummatory behavior was measured. One group was given only the adulterated food; the second group began with the preferred food and then was shifted to the adulterated food (the conventional way of looking for successive negative contrast). In the final stage of the experiment, the unshifted group continued to be given only the adulterated food, and the shifted group was given the preferred food on some days and the adulterated food on others.

\section{Method}

Subjects. The subjects were $2410-\mathrm{cm}$ goldfish, all experimentally naive. They were maintained in individual compartments formed by partitioning larger tanks; their water was continuously filtered and recirculated with partial replacement.

Apparatus. For the measurement of consummatory behavior, each animal was transferred to a dark chamber with inflow of water from the living tanks and outflow directly to the drains. (There were two such chambers in which 2 subjects could work concurrently). Against one wall of the chamber was a circular target of lightdiffusing Plexiglas, $4 \mathrm{~cm}$ in diameter, which could be illuminated by one of several different colored lamps. At the center of the target was a small Plexiglas cup into which liquid food could be deli- vered automatically by a syringe pump. The target was fixed to a rod, the other end of which was attached to a strain gauge mounted above the chamber. Contact with the target was detected by an integrating circuit that recorded the contact and caused a drop $(10 \mu \mathrm{l})$ of food to be delivered to the cup. The opening through which water left the chamber was directly below the target, and any excess food was carried away immediately in the outflow.

Procedure. The subjects were divided at random into two groups of 12 , a contrast group and a control group. Each animal was given a series of 30-min daily sessions in the apparatus. After a few minutes of darkness, each session began with illumination of the target by a white lamp and the delivery of a drop of food. As the animal took the food, it made contact with the target, which operated the pump and so produced another drop of food. In early sessions, the pump also was operated by a timer once each minute in order to attract the animal to the target, a practice that was discontinued as soon as the animal began to respond at a greater rate. At the end of $30 \mathrm{~min}$, the target light was turned off and the animal was taken back to its living enclosure. In the late afternoon, all animals were postfed with pellets of dry food.

The first phase of the experiment consisted of 24 daily sessions during which the contrast group was given a preferred food (made by blending $30 \mathrm{~g}$ of Tetramin flakes with $4.5 \mathrm{~g}$ of powdered tragacanth in $300 \mathrm{ml}$ of water), and the control group was given the same food blended with enough quinine sulfate $(.5 \mathrm{mg} / \mathrm{ml}-\mathrm{a}$ value selected in pilot experiments) to make it distinctly less attractive. In the second phase of the experiment (Sessions 25-40), the procedure was the same except that both groups were given the adulterated food. In the third phase of the experiment (Sessions 41-55), the control group continued to be given the adulterated food, whereas the contrast group was given the adulterated food in some sessions $(42,43,47,48,51,54,55)$ and the preferred food in the rest. The intermixing of experience with the two foods for the contrast group, reminiscent of the simultaneous contrast procedure, was designed to enhance frustration when the adulterated food was presented. Repeated encounters with two sucrose solutions of different concentrations on separate days has produced strong and sustained contrast in the consummatory behavior of rats (Flaherty, Becker, \& Checke, 1983).

\section{Results}

In the first phase of the experiment, as Figure 1 shows, the rate at which the animals took food increased progressively. The contrast animals responded much more rapidly for the preferred food than did the control animals for the adulterated food, with no overlap between the two groups. In the first panel of Figure 2, the terminal withinsessions performance of the two groups, based on the

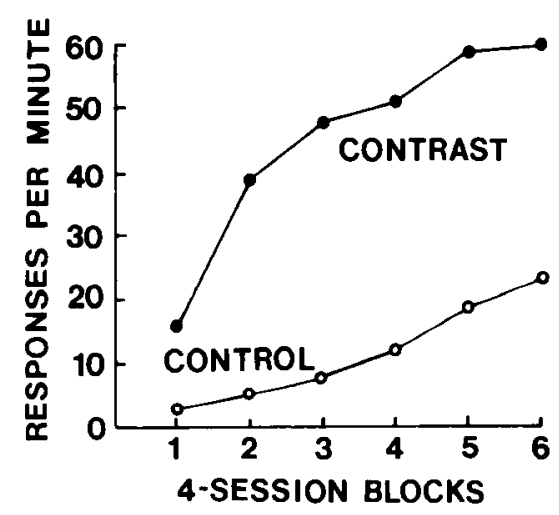

Figure 1. Responding of the contrast animals for the preferred food and of the control animals for the adulterated food in the first phase of Experiment 1. 

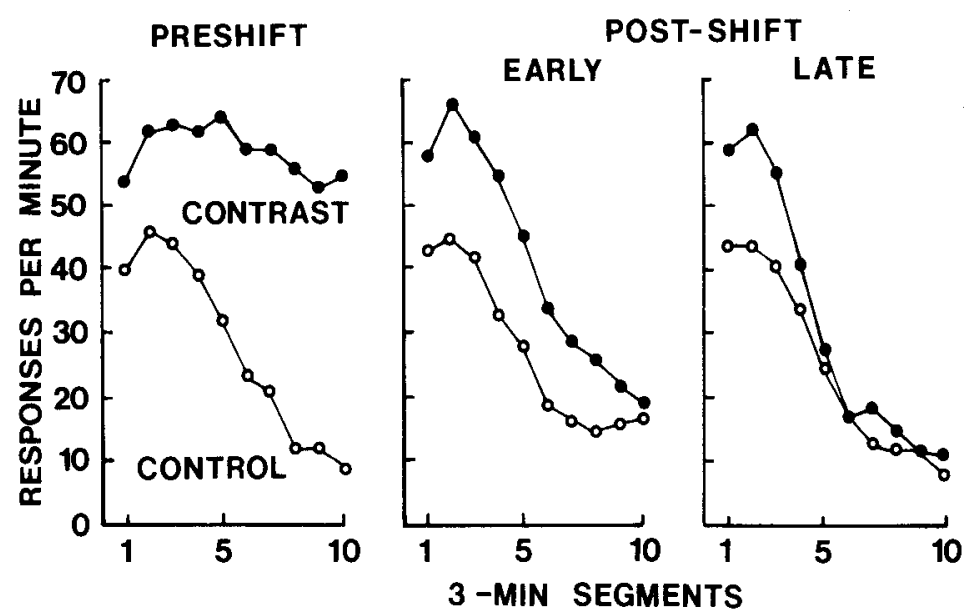

\title{
STAGE 3
}

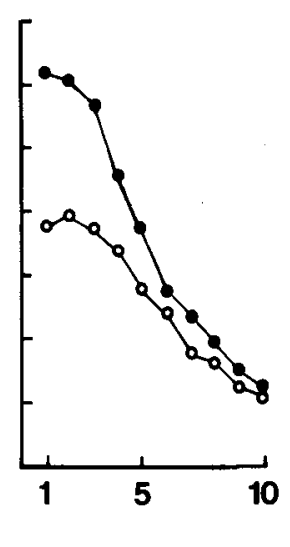

\begin{abstract}
Figure 2. Pooled within-session plots of consummatory responding by the two groups in Experiment 1 . In the preshift phase, the contrast animals were given the preferred food and the control animals were given the adulterated food. In the postshift phase, both groups were given the adulterated food. In the third stage of the experiment, the contrast animals were given the preferred food in some sessions and the adulterated food in others, while the control animals were given only the adulterated food; the points plotted are for sessions in which both groups were given the adulterated food.
\end{abstract}

pooled data for Sessions 23 and 24 , is plotted in terms of mean rate of responding in the 10 successive 3 -min segments. The curves show that there was less response for the adulterated food than for the preferred food at the outset of each session, although the main difference is that response for the adulterated food fell off more quickly in the course of the session than did response for the preferred food. (Goldfish continue to be attracted to the preferred food for long periods, seemingly by its sensory properties, although after a time the food is not swallowed, but expelled through the gill covers.)

When, in the second phase of the experiment, the contrast group was shifted to the adulterated food, it responded more, not less, than the control group. Pooled within-sessions curves for the first two postshift sessions (25 and 26) are plotted in the second panel of Figure 2. Consummatory responding in both groups declined in the course of each session, but was higher throughout in the contrast group. In Sessions 29-33, the performance of the groups was much the same, but then a difference appeared again which persisted through the remaining sessions of the second phase. The contrast animals responded more rapidly than the control animals only in the first portion of each session, as illustrated in the third panel of Figure 2, which presents the pooled within-sessions curves for the last two sessions ( 39 and 40 ) of the second phase. Analysis of variance for the data from all 16 sessions of the second phase yields no significant groups effect $[F(1,22)=1.14, p=.29]$, but significant interactions of groups $\times$ sessions $[F(15,330)=2.68, p=.0007]$ and of groups $\times(3-\mathrm{min})$ segments $[\mathrm{F}(9,198)=4.92, \mathrm{p}<$ .00011 .

In the third phase of the experiment, the contrast group continued, despite repeated experience with the preferred food, to respond more for the adulterated food than did the control group. The pooled within-sessions perfor- mance of the two groups for the seven sessions in which both were given the adulterated food is shown in the fourth panel of Figure 2. The groups effect is not significant $[\mathrm{F}(1,22)=2.62, \mathrm{p}=.12]$, but there is a highly reliable groups $\times$ segments interaction $[\mathrm{F}(9,198)=4.68, \mathrm{p}<$ $.0001]$. The data of this experiment, then, provide no evidence that experience with the preferred food reduces the acceptability of the less preferred food. On the contrary, experience with the preferred food tends to increase responding for the less preferred food at the outset, at least, of each exposure to it.

\section{EXPERIMENT 2}

In this experiment, we again studied the effect of experience with a preferred food on consummatory responding for a less preferred food, but we used a newer design that demonstrates what has been called "anticipatory" negative contrast in rats (Flaherty \& Checke, 1982). In this design, the contrast group is given a preferred food soon after a less preferred food, while the control group is given only the less preferred food, with the result that there is less responding for the less preferred food in the contrast group than in the control group. According to frustration theory, of course, all negative contrast has an anticipatory basis, but what the term emphasizes in this context is the finding that the interval between presentations of the two foods to the contrast group is critical, and the rat data do, in fact, show that the effect decreases in magnitude, although by no means entirely, as the interval increases from 1 to $30 \mathrm{~min}$. The interpretation is that the pairing of the two foods produces a particularly strong anticipation of the preferred food in the presence of the less preferred food and so enhances dissatisfaction with the less preferred food. The design also has the advantage of exposing the contrast animals to both foods in each 
session and in a sequence that rules out any short-term, nonassociative effects of exposure to the preferred food on the acceptability of the less preferred food. As has already been noted, such effects are not ruled out in the conventional massed-trials experiments that show simultaneous contrast.

\section{Method}

Subjects. The subjects were 14 naive goldfish, $10 \mathrm{~cm}$ long, that were maintained in the same way as those of the previous experiment.

Apparatus. The apparatus was the same as that used in the previous experiment.

Procedure. All subjects were pretrained with the adulterated food (the same as the preferred food but with quinine sulfate added, $.5 \mathrm{mg} / \mathrm{ml}$ ). First there were 16 daily sessions, each $15 \mathrm{~min}$ in duration, with the procedure the same as previously described. Then there were 21 sessions whose duration was reduced to $5 \mathrm{~min}$. In the final phase of the experiment ( 24 sessions), the animals were divided into two groups matched for rate of responding in the pretraining sessions. In each session for both groups, there were two 5-min feeding periods separated by a 10 -sec interval in darkness. During both feeding periods, the color of the target light was not white, as it had been in the pretraining, but was red for half the animals in each group and green for the rest. (The colors were balanced in preparation for subsequent work whose nature is not relevant here.) For both groups of animals, the adulterated food was given in the first feeding period. In the second period, the control group was given the adulterated food again, and the contrast group was given the preferred food.

\section{Results}

The rate at which the animals responded to the adulterated food increased progressively in pretraining, stabilizing in the 5-min sessions at a rate of about 45 responses/ min (the same as at the outset of the longer feeding periods used in Experiment 1). The performance of the two groups in the final phase of the experiment is plotted in Figure 3 in terms of rate of responding for the adulterated food, which was given to both groups in the first 5-min feeding period of each session. One interesting feature of the results is that the change in target color (from the white used in pretraining) produced a marked decrement in rate of responding for the adulterated food. The subsequent recovery was very gradual, and was slower in the control group than in the contrast group. In relation

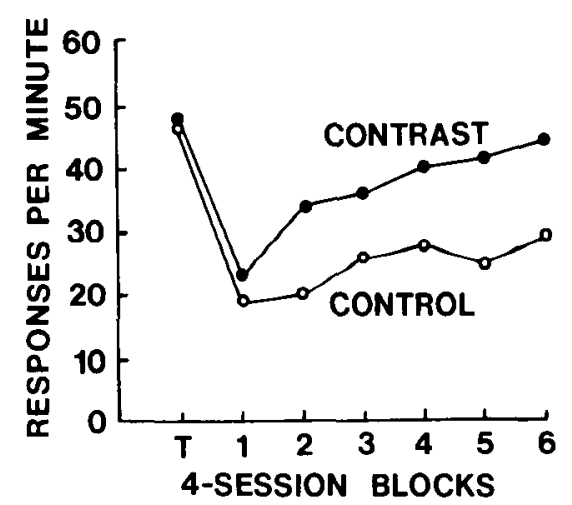

Figure 3. Responding for the adulterated food by the two groups at the end of pretraining (T) and in the final phase of Experiment 2. to our search for evidence of negative contrast, the important result is that the contrast group responded more, not less, for the adulterated food than did the control group. Impressions from the curves are confirmed by analysis of variance, which yields a significant groups effect $[F(1,13)=12.68, p=.0039]$, a significant sessions effect $[F(23,276)=4.36, p<.0001]$, and a significant groups $\times$ sessions interaction $[\mathrm{F}(23,276)=2.02, \mathrm{p}=$ $.0045]$.

\section{DISCUSSION}

The consummatory responding of goldfish for a less preferred food is not suppressed by prior experience with a preferred food, but tends, on the contrary, to be enhanced by that experience. In Experiment 1, consummatory responding for the adulterated food developed very gradually in the control animals, which were exposed to it from the outset, but the contrast animals, which were trained with the preferred food, took the adulterated food readily on their first exposure to it-even more readily than did the control animals. In subsequent sessions with the preferred food given on some days and the adulterated food on other days, the contrast animals continued to take the adulterated food more readily than did the control animals, which were given only the adulterated food. In Experiment 2, there was again more responding to the adulterated food in the contrast group (for which exposure to the adulterated food at the start of each daily session soon was followed by exposure to the preferred food) than in the control group (for which the initial exposure to the adulterated food was followed instead by further exposure to the same food). These results are directly opposite to those of analogous experiments with rats, in which the preferred and less preferred foods were sucrose solutions of different concentration. The possibility that our use of quinine is somehow responsible for the difference is ruled out by a recent experiment with rats (personal communication from C. F. Flaherty) in which the addition of quinine $(30 \mathrm{mg} / 100 \mathrm{ml})$ to $40 \%$ sucrose was found to produce a successive contrast effect as large as, and more enduring than, that produced by the usual shift from $32 \%$ to $4 \%$ sucrose.

Although the response recorded in the present experiments is fairly characterized as consummatory, it clearly is not a response to food alone. The precipitous decline in the performance of both groups when the color of the target was changed in Experiment 2 shows control by color, which perhaps should not be surprising in view of previous work on autoshaping (Woodard \& Bitterman, 1974). What may be more surprising is that there was no such decline in the performance of the contrast animals in the second phase of Experiment 1, when they encountered the adulterated food for the first time. Their response to the new food fell off gradually in the course of the session, as did that of the control animals; but in the first 10 min of the session the contrast animals responded very rapidly, giving no hint of the generalization decrement 
that might have been expected (quite apart from the possibility of frustration) on the assumption that they were responding to the food as to the lighted target. The fact that the contrast animals responded even more rapidly for the adulterated food than did the controls in both experiments suggests that food functioned primarily as reinforcement, and that the preferred food was more reinforcing. It may be, then, that the procedure employed here was actually not much different from instrumental training in highly massed trials, but even so, the conditions for the appearance of contrast should have been especially favorable.

Although there seems to be no way to rule out the possibility that there are as-yet-undiscovered circumstances in which goldfish would show successive contrast, or the possibility that the negative results are due to the weakness, rather than the absence, in goldfish of whatever mechanism produces the phenomenon in rats (see also Mackintosh, 1971), we are inclined on the basis of the present results to give more weight to other possibilities (Bitterman, 1975). One is that goldfish, responding in anticipation of a preferred food, are nevertheless undisturbed (not frustrated) when they encounter a less preferred food. Another is that responding in goldfish is controlled not by its remembered outcomes, but only by its immediate sensory antecedents in simple Pavlovian or Thorndikian fashion. Although hardly sufficient to the mammalian data, the assumption that conditioned stimuli directly instigate responding is difficult to dispense with entirely (cf. Mackintosh, 1983), and it is conceivable that what we are seeing in descendants of older vertebrate lines (goldfish, toads, and painted turtles) and in very young rats is direct instigation as yet unmodulated by associatively reinstated representations of past events. The emphasis, of course, is on associative reinstatement, which should be distinguished carefully from sensory carryover (Couvillon \& Bitterman, 1981).

Complementing the results of what is by now a substantial array of experiments that fail to demonstrate control of performance by remembered reward in goldfish are some negative results on control by remembered nonreward, as, for example, the absence of the partial reinforcement effect in widely spaced trials (Schutz \& Bitterman, 1969; see also the results for African mouthbreeders, Longo \& Bitterman, 1960). The spacedtrials partial reinforcement effect in rats, which occurs only with large reward (Gonzalez \& Bitterman, 1969; Hulse, 1958; Wagner, 1961), seems to depend both on memory of reward and on memory of nonreward (whether conceived as reinstatement or as feedback from conditioned frustration), the former responsible for precipitous extinction in the consistently rewarded group, and the latter for less rapid extinction in the inconsistently rewarded group. Goldfish trained in massed trials with special procedures employed to randomize sensory carryover fail also to show patterning effects (Couvillon, 1984) that seem in pigeons to reflect control by memory of nonreward (Couvillon, Brandon, Woodard, \& Bitterman, 1980).

\section{REFERENCES}

Bitterman, M. E. (1975). The comparative analysis of learning. Science, 188, 699-709.

BoWER, G. H. (1961). A contrast effect in differential conditioning. Journal of Experimental Psychology, 62, 196-199.

Burns, R., Woodard, W. T., Henderson, T. B., \& Bitterman, M. E. (1974). Simultaneous contrast in the goldfish. Animal Learning \& Behavior, 2, 97-100.

Chen, J., Gross, K., \& Amsel, A. (1981). Ontogeny of successive negative contrast and its dissociation from other paradoxical reward effects in preweanling rats. Journal of Comparative \& Physiological Psychology, 95, 146-159.

Couvillon, P. A. (1984). Performance of goldfish in patterned sequences of rewarded and nonrewarded trials. Journal of Comparative Psychology, 98, 333-344.

Couvillon, P. A., \& Bitterman, M. E. (1981). Analysis of alternation patterning in goldfish. Animal Learning \& Behavior, 9, 169-172.

Couvillon, P. A., Brandon, S. E., Woodard, W. T., \& BitterMAN, M. E. (1980). Performance of pigeons in patterned sequences of rewarded and nonrewarded trials. Journal of Experimental Psychology: Animal Behavior Processes, 6, 137-154.

Elliott, M. H. (1928). The effect of change of reward on the maze performance of rats. University of Califormia Publications in Psychology, 4, 19-30.

FlaherTy, C. F. (1982). Incentive contrast: A review of behavioral changes following shifts in reward. Animal Learning \& Behavior, 10, 409-440.

Flaherty, C. F., Becker, H. C., \& Checke, S. (1983). Repeated successive contrast in consummatory behavior with repeated shifts in sucrose concentration. Animal Learning \& Behavior, 11, 407-414.

Flaherty, C. F., CheCKe, S. (1982). Anticipation of incentive gain. Animal Learning \& Behavior, 10, 177-182.

Gonzalez, R. C., Bitterman, M. E. (1969). Spaced-trials partial reinforcement effect as a function of contrast. Journal of Comparative \& Physiological Psychology, 67, 94-103.

Gonzalez, R. C., Ferry, M., \& Powers, A. S. (1974). The adjustment of goldfish to reduction of magnitude of reward in massed trials. Animal Learning \& Behavior, 2, 23-26.

Gonzalez, R. C., Potts, A., Pitcoff, K., \& Bitterman, M. E. (1972). Runway performance of goldfish as a function of complete and incomplete reduction in amount of reward. Psychonomic Science, 27, 305-307.

Gonzalez, R. C., \& Powers, A. S. (1973). Simultaneous contrast in goldfish. Animal Learning \& Behavior, 1, 96-98.

HuLSE, S. H., JR. (1958). Amount and percentage of reinforcement and duration of goal confinement in conditioning and extinction. Journal of Experimental Psychology, 56, 48-57.

LoNGo, N. \& BitTerman, M. E. (1960). The effect of partial reinforcement with spaced practice on resistance to extinction in the fish. Joumal of Comparative \& Physiological Psychology, 53, 169-172.

Lowes, G., \& BitTerman, M. E. (1967). Reward and learning in the goldfish. Science, 157, 455-457.

Mackintosh, N. J. (1971). Reward and aftereffects of reward in the learning of goldfish. Journal of Comparative \& Physiological Psychology, 76, 225-232.

MaCKINTOSH, N. J. (1983). Conditioning and associative leaming. Oxford: Clarendon Press.

Pert, A., \& Bitterman, M. E. (1970). Reward and learning in the turtle. Learning \& Motivation, 1, 121-128.

Pert, A., \& Gonzalez, R. C., (1974). Behavior of the turtle (Chrysemys picta picta) in simultaneous, successive, and behavioral contrast situations. Journal of Comparative \& Physiological Psychology, 87, 526-538.

Rashotre, M. E. (1979). Reward training: Contrast effects. In M. E. Bitterman, V. M. LoLordo, J. B. Overmeir, \& M. E. Rashotte (Eds.), Animal learning: Survey and analysis (pp. 195-240). New York: Plenum Press.

Schmajuk, N. A., Segura, E. T., \& Ruidiaz, A. C. (1981). Reward downshift in the toad. Behavioral \& Neural Biology, 33, 519-523. 
Schutz, S. L., \& Bitrerman, M. E. (1969). Spaced-trials partial reinforcement and resistance to extinction in the goldfish. Journal of Comparative \& Physiological Psychology, 68, 126-128.

Stanton, M., AMSEl, A. (1980). Adjustment to reward reduction (but no negative contrast) in rats 11,14 , and 16 days of age. Journal of Comparative \& Physiological Psychology, 94, 446-458.

Stanton, M. Lobaugh, N., \& Amsel, A. (1984). Age of first appearance of simultaneous and successive negative contrast in infant rats. Journal of Experimental Psychology: Animal Behavior Processes, 10, 376-389.
WAGNER, A. R. (1961). Effects of amount and percentage of reinforcement and number of acquisition trials on conditioning and extinction. Journal of Experimental Psychology, 62, 234-242.

Woodard, W. T., \& Bitterman, M. E. (1974). Autoshaping in the goldfish. Behavior Research Methods \& Instrumentation, 6, 409-410.

(Manuscript received March 25, 1985; revision accepted for publication August 22, 1985.) 